\title{
De Novo Assembly of Lucina pectinata Genome using lon Torrent Reads
}

\author{
Ingrid M. Montes-Rodríguez \\ Comprehensive Cancer Center \\ University of Puerto Rico \\ P.O. BOX 363027 \\ San Juan, PR 00936 \\ 787-772-8300 \\ ingrid.montes@gmail.com
}

\author{
Carmen L. Cadilla \\ University of Puerto Rico \\ Medical Sciences Campus \\ P.O. Box 365067 \\ San Juan, PR 00936 \\ 787-758-2525 \\ carmen.cadilla@upr.edu
}

\author{
Ricardo González-Méndez \\ University of Puerto Rico \\ Medical Sciences Campus \\ P.O. Box 365067 \\ San Juan, PR 00936 \\ 787-758-2525 \\ ricardo.gonzalez7@upr.edu
}

\author{
Juan Lopéz-Garriga \\ University of Puerto Rico \\ Mayaguez Campus \\ PO BOX 9019, \\ Mayaguez 00681-9019 \\ 787-832-4040 \\ Juan.lopez16@upr.edu
}

\author{
Alexander Ropelewski \\ Pittsburgh Supercomputing Center \\ 300 South Craig Street \\ Pittsburgh PA 15213 \\ 1-412-268-4960 \\ ropelews@psc.edu
}

\begin{abstract}
Lucina pectinata is a bivalve that lives in sulfide-rich environments and houses intracellular sulfide oxidizing endosymbiont. This organism is an ideal model to understand adaptive mechanisms and chemoautotrophic endosymbiosis in organisms living in sulfide-rich environments. However, only three hemoglobins have been completely characterized at protein and gene level leaving a gap in understanding the biology of this organism. In this work, we produced draft genomic assemblies with data produced by the Ion Proton Next Generation Sequencing System using both the MIRA4 and SPAdes assemblers. We compare and contrast these draft assemblies using metrics such as N50, total assembled length, number of predicted genes and other measures. We conclude that de novo assembly of eukaryotic organisms with NGS data from the Ion technology family remains complicated and may benefit from the use of multiple genome assemblers. .
\end{abstract}

\section{CCS Concepts}

- Computing methodologies $\rightarrow$ Massively parallel and high-performance simulations.

- Applied computing $\rightarrow$ Life and medical sciences $\rightarrow$ Computational biology $\rightarrow$ Computational Genomics.

This work is licensed under a Creative Commons Attribution-NonCommercial-NoDerivs International 4.0 License.

PEARC17, July 09-13, 2017, New Orleans, LA, USA (C) 2017 Copyright is held by the owner/author(s). ACM ISBN 978-1-4503-5272-7/17/07. http://dx.doi.org/10.1145/3093338.3093362

\section{Keywords}

Bioinformatics; Genome Assembly; MIRA; SPAdes; Ion semiconductor sequencing; Next Generation Sequencing (NGS); Lucina pectinata; High Performance Computing (HPC).

\section{ACM Reference format:}

I. M. Montes-Rodríguez, C. L. Cadilla R. González-Méndez, J. LópezGarriga and A. Ropelewski. 2017. De Novo Assembly of Lucina pectinata Genome using Ion Torrent Reads. Practice and Experience in Advanced Research Computing 2017 Proceedings, New Orleans, LA, USA, July 0913, 2017 (PEARC17), 6 pages.

DOI: $10.1145 / 3093338.3093362$

\section{INTRODUCTION}

Hydrogen sulfide (H2S) has been considered a metabolic poison, since at nanomolar to millimolar concentrations, it adversely affects the cytochrome aa3 unit of the cytochrome c oxidase complex [1], oxygen transport proteins, cellular structures, and consequently the physiological functions of organisms [2]. However, there is a vast variety of marine invertebrates inhabit ecosystems characterized by displaying high $\mathrm{H} 2 \mathrm{~S}$ concentration, such as hydrothermal vents [3], [4], cold seeps [5], and muds [6]. The clam Lucina pectinata (L. pectinata) lives in anoxic sulfide-rich muds in the Southwestern coast of Puerto Rico and throughout the Caribbean Sea and houses intracellular symbiotic bacteria that need to be supplied with both hydrogen sulfide $\left(\mathrm{H}_{2} \mathrm{~S}\right)$ and oxygen $\left(\mathrm{O}_{2}\right)$. L . pectinata is an ideal model to understand adaptive mechanisms and chemoautotrophic endosymbiosis in organisms living in sulfide-rich environments. So far, three different hemoglobins have been identified in L. pectinata: hemoglobin I (HbI), a sulfide-reactive protein, and hemoglobin II (HbII) and III (HbIII), which are oxygen-reactive proteins. It is believed that the main biological function of $\mathrm{HbI}$ is to transport $\mathrm{H} 2 \mathrm{~S}$ within the mollusk and to make it accessible to the chemoautotrophic bacteria, whereas $\mathrm{HbII}$ and $\mathrm{HbIII}$ are responsible for $\mathrm{O} 2$ transport. 
Besides these three Hbs, only partial protein sequences are known for cytochrome c oxidase subunit I, histone $\mathrm{H} 3$ and cytochrome $\mathrm{B}$. However, so far, no other proteins have been characterized in this clam, leaving a big gap in understanding the biology of this organism.

Nowadays, advances in sequencing technologies such as NextGeneration Sequencing (NGS) and the accompanying bioinformatics advances allow investigators to obtain invaluable basis for studying every aspect of an organism's biology. The Ion platforms (Ion PGM ${ }^{\mathrm{TM}}$ and Ion Proton ${ }^{\mathrm{TM}}$ Sequencers) perform massively parallel sequencing using semiconductor sequencing technology [7]. In this technology, the machine detects a change in $\mathrm{pH}$ after a proton and a pyrophosphate $(\mathrm{Pi})$ are released when a nucleotide is incorporated into the DNA molecules by the polymerase. The machine flows one nucleotide at a time through the chip surface, if it is not the correct nucleotide, no voltage change will be detected; if there are 2 nucleotides added, a double voltage change will be detected [7]-[9]. An advantage of the Ion Proton sequencer is that it produces rapid sequencing results for low costs. Like the Roche 454 sequencer, (and the newly introduced Oxford Nanopore MinION ${ }^{\mathrm{TM}}$ sequencer) the dominate errors produced from the sequencer family are insertion/deletion errors. This contrasts to the Illumina family of sequencers where substitutions are the typical errors produced [10].

Partially due to the error profile and the difficulty producing matepaired libraries, genomic use of Ion sequencing technology is commonly used for the de novo sequencing of viral and microbial genomes or to produce prokaryotic or eukaryotic reference based assemblies. The use of Ion technology to produce de novo assemblies of large eukaryotic genomes is rare. We have only been able to identify one publication in Pubmed that assembled Ion data for a large Eucaryotic Genome de novo [11]. This paper outlines our experience assembling and annotating Ion eukaryotic genomic data de novo.

\section{METHODS}

\subsection{Biological}

Ethics statement: Juvenile L. pectinata clams were purchased from a local fisherman in the town of Cabo Rojo, PR. L. pectinata is not an endangered or protected species, hence, no specific permits were required to obtain the clams since they are a local food item.

\subsubsection{DNA isolation}

DNA from L. pectinata was extracted from the Ctenidia tissue using the Omega Biotek E.Z.N.A. Mollusk DNA Kit following the instructions from the manufacturer.

\subsubsection{Ion Proton Library preparation}

Library for Ion Proton sequencing was created using the IonPlus Fragment Library kit from Life Technologies, according to the Ion Xpress $^{\mathrm{TM}}$ Plus gDNA Fragment Library Preparation protocol (Publication Number MAN0009847). The emulsion PCR and enrichment of the library was carried out in the Ion OneTouch ${ }^{\mathrm{TM}}$ 2 System and in the Ion OneTouch ${ }^{\mathrm{TM}}$ ES System, respectively, using the Ion PI Template OT2 200 Kit v3 from Life
Technologies following the Ion PI ${ }^{\mathrm{TM}}$ Template OT2 200 Kit v3 User Guide (Publication Number MAN0009133). The sample was run in the Ion Proton ${ }^{\mathrm{TM}}$ Sequencer using the Ion PI ${ }^{\mathrm{TM}}$ Chip V2 from Life Technologies. The sample was prepared using the Ion PI ${ }^{\mathrm{TM}}$ Sequencing 200 Kit v3 following the Ion $\mathrm{PI}^{\mathrm{TM}}$ Sequencing $200 \mathrm{Kit}$ v3 User Manual (Publication Number MAN0009136).

To create the draft de novo assemblies, an unpaired low-coverage dataset was selected according to the output and quality of data available.

\subsection{Computational}

Computational work was performed on the Pittsburgh Supercomputing Center's Blacklight [12] and Bridges [13] systems, high-performance computing systems available through the National Science Foundation (NSF) XSEDE Program[14]. Blacklight (now decommissioned) was an SGI UV 1000 cc-NUMA shared-memory system comprised of 256 blades. Each blade shared 128 GB of local memory, and held two Intel Xeon X7560 (Nehalem) eight-core processors, for a total of 4,096 cores and 32 TB across the whole system. Bridges consists of tiered, largeshared-memory resources with nodes having $12 \mathrm{~TB}, 3 \mathrm{~TB}$, and $128 \mathrm{~GB}$ each, dedicated nodes for database, web, and data transfer, high-performance shared and distributed data storage, Hadoop acceleration, and an OmniPath interconnection network. Bridges $12 \mathrm{~TB}$ nodes contain 16x Intel Xenon E7-8880 processors, 3TB nodes contain either $4 \mathrm{x}$ Intel Xenon E5-8860 or 4x Intel E7-8870 processors and 128TB nodes contain 2x Intel Haswell E5-2695 processors.

\subsubsection{Data Preparation}

Reads were first examined for quality using the FastQC tool [15]. The data used for assembly was $12.4 \mathrm{~Gb}$ of single-end sequence read data (the sequencer reads the fragment from only one end to the other) and contained a total of 80,030,719 reads with a mean length of $155 \mathrm{bp}$. The mean sequence quality score for the read data was 21. We then trimmed and filtered the data using the Sickle Windowed Adaptive Trimming Tool. We used a quality score threshold of 15 and discarded reads that were shorter than 75 bases in length. After this filtering, the data set contained approximately 59 million reads and 10 billion bases, yielding an average read length of about 170 bases.

\subsubsection{Assembly}

Draft assemblies of the trimmed data were produced with both MIRA [16] and SPAdes [17]. MIRA version 4.0.2 was run using the parameter set IONTOR_SETTINGS. MIRA estimated the coverage of the data to be at approximately 6.3X coverage. Spades version 3.8.1 was run using the -iontorrent flag and $\mathrm{K}$ values of 21, 33 and 55.

\subsubsection{Gene and RNA Prediction}

Gene predictions were produced using the GlimmerHMM software suite [18]. The gene prediction model used was produced from the closely-related mollusk genome of Lottia gigantea (Giant owl limpet). Various RNA prediction programs/models were used with the default parameters. 


\subsubsection{Gene Annotation}

Predicted genes were searched against UniProt Swissprot and Trembl [19] release 2-15-2017 and the UniProt Lottia gigantea (Giant owl limpet) proteome (UP000030746). Searches against these databases were performed using the Diamond program [20] in BlastX mode with the BLOSUM62 Matrix, a gap open penalty of -11 and a gap extend penalty of -1 .

Gene ontology (GO) analysis was performed for both assemblies with the Software Tool for Researching Annotations of Proteins (STRAP), which obtains gene ontology GO terms associated with proteins using the freely accessible UniProtKB and EBI GOA databases [21].

\subsubsection{Mitochondrial genome}

TBLASTN searches were performed using the 13 protein sequences encoded by the Solemya velum mitochondrial genome (Accession NC_017612.1) as query sequences against the MIRA and SPAdes assemblies. The identified MIRA and SPAdes scaffolds were re-scafolded using CAP3 program[22]. The ARAGORN and INFERNAL cmscan programs were used to predict tRNA and rRNA sequences in the assembled scaffolds[23], [24].

\section{RESULTS}

The MIRA assembly was run using 16 cores and 384 GB (48 cores) of memory on Blacklight. The assembly took 45 days to complete $(\sim 52,000 \mathrm{SU})$ and generated $889 \mathrm{~GB}$ of data files. The SPAdes draft assembly, which was run on a Bridges 3TB node, used 32 cores and took approximately one day to complete. The SPAdes program reported using a peak of $207 \mathrm{~GB}$ of memory. The program generated about $20 \mathrm{~GB}$ of data files.

Table 1. Contig summary of the L. pectinata draft genome assembly

\begin{tabular}{lrr}
\hline \multicolumn{1}{c}{ Metric } & \multicolumn{1}{c}{ MIRA } & \multicolumn{1}{c}{ SPAdes } \\
\hline Number of Scaffolds & $2,092,332$ & $1,120,359$ \\
\hline Scaffolds over 10k bases & 9 & 38 \\
\hline Scaffolds over 1k bases & 339,458 & 162,888 \\
\hline Total Bases Assembled & $1,339,356,593$ & $539,065,997$ \\
\hline $\begin{array}{l}\text { Length assembled } \\
\text { Scaffolds over 10k bases }\end{array}$ & 114,506 & $1,120,321$ \\
\hline $\begin{array}{l}\text { Length assembled } \\
\text { Scaffolds over 1k bases }\end{array}$ & $52,669,480$ & $262,164,089$ \\
\hline Largest Scaffold & 19,026 & 26,708 \\
\hline N50 & 806 & 973 \\
\hline GlimmerHMM exons & 73,521 & 31,927 \\
\hline GlimmerHMM mRNA & 65,984 & 27,302 \\
\hline $\begin{array}{l}\text { mRNA with UniProt } \\
\text { matches }\end{array}$ & 8,906 & 3,269 \\
\hline Unique UniProt matches & & \\
\hline $\begin{array}{l}\text { mRNA with Lotgi } \\
\text { proteome matches }\end{array}$ & 6,243 & 2,946 \\
\hline Unique proteome Matches & 4,415 & 2,075 \\
\hline
\end{tabular}

A summary comparing the results of the MIRA and SPAdes assemblies along with relative assembly quality metrics are shown in Table 1 above. In addition to these metrics, we used the STRAP program to analyze the annotation results in terms of gene ontology terms. The STRAP program, divided the annotated sequences in 24 functional groups within the three main gene ontologies: cellular component, molecular function and biological processes, see Figure 1.

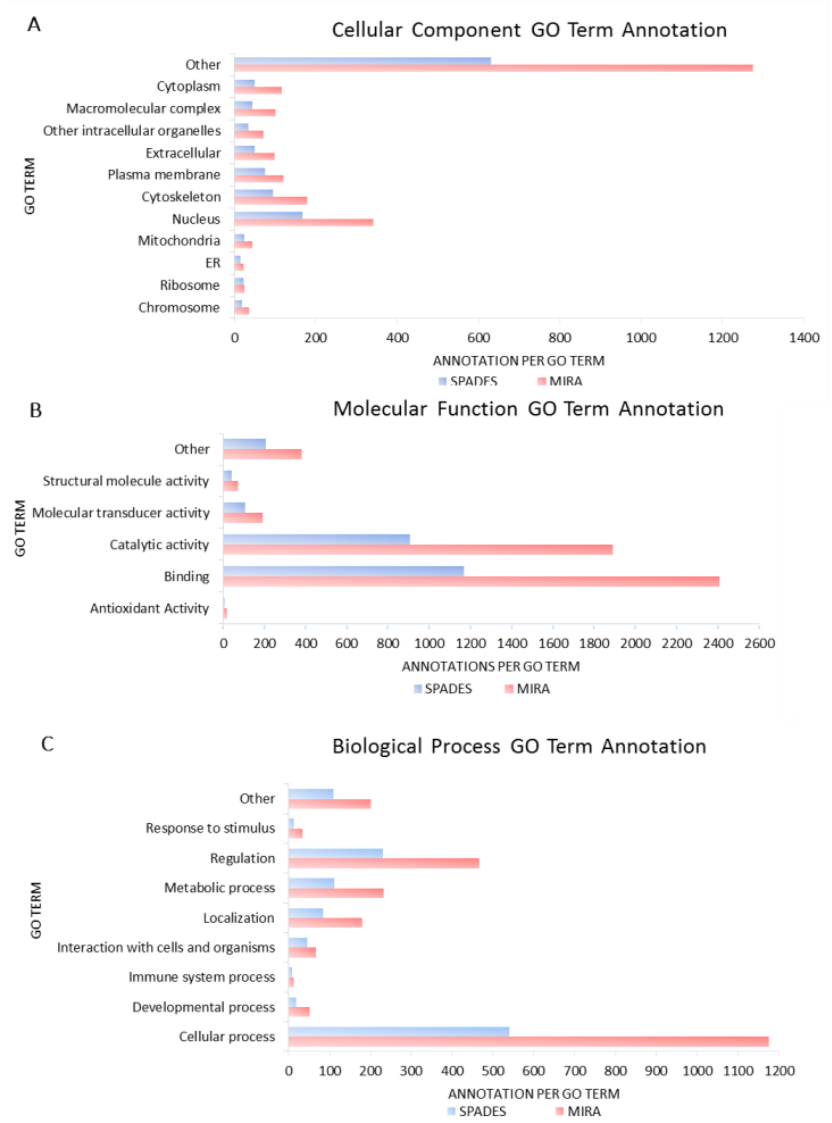

Figure 1. Graphical representation of the Gene Ontology classification using the STRAP program. The results are summarized in the three main Gene Ontology categories: A) Cellular Component; B) Molecular Function; and C) Biological Process. The $y$-axis indicates the gene ontologies categories and the $x$-axis indicates the number of genes in a category

\section{DISCUSSION}

Algorithmically, the MIRA assembler models the assembly problem as a Hamiltonian problem, relying on the comparison of the reads to produce an overlap graph. Since Hamiltonian path problem is NP complete, it is unsurprising to see the large run times with our data. SPAdes represents the problem as an Eulerian graph problem, breaking down the sequence data into small overlapping words of a fixed size. These fixed-size words are used to construct de Bruin graphs with the path through the graphs representing the assembled genome. 
Because NGS data is subject to experimental errors and limited lengths, both heuristic and statistical procedures are incorporated into assembly codes to correct error-prone reads, eliminate false paths and untangle the graphs into large un-broken strings of genomic data. These procedures vary from method-to-method and while typically helpful, they are imperfect and can sometimes introduce errors into an assembly. Furthermore, Ion data is particularly subject to homopolymer sequencing errors [25] which can complicate the assembly process.

\subsection{Assembly Analysis}

\subsubsection{N50, number of contigs and assembled length}

The N50 statistic is one of the most widely used statistic for describing the quality of a genome assembly. N50 is calculated by first ordering every contig by length from longest to shortest. Next, starting from the longest contig, the lengths of each contig are summed, until this running sum equals one-half of the total length of all contigs in the assembly. The contig N50 of the assembly is the length of the shortest contig in this list [26]. From this statistical perspective, the SPAdes assembly has a higher N50 value approximately $1.2 \mathrm{x}$ larger that the MIRA assembly. The small average contig size, as indicated by the N50 metric was expected due to the repetitive nature of the genome and the use of single-end reads [27], [28].

Overall, the MIRA assembly generated more total contigs than the SPAdes assembly which led to a larger overall assembled size of $1.3 \mathrm{~Gb}$. SPAdes produced a total assembled length that was approximately $5 \mathrm{x}$ shorter than the MIRA assembler. Given the estimated size of $L$. pectinata's genome is about $1.6 \mathrm{~Gb}$ [29] the MIRA assembler appears to have assembled more bases of the genome than SPAdes. However, looking at the total number bases assembled into large contigs, SPAdes appears to have performed better.

\subsubsection{Gene Prediction and Annotation}

Due to the larger total assembled lengths, GlimmerHMM predicted on average between two and three times more potential coding regions from the MIRA assembly than the SPAdes assembly.

When looked at in terms of numbers of database sequences matched, the MIRA assembly contains both more overall matches and more unique matches in both the UniProt database and the Lottia gigantea proteome. The database search against the Lottia gigantea proteome found 1.8x times more significant unique entry matches with the MIRA assembly than with the SPAdes assembly. This indicates that the MIRA assembly contains unique features of the clam genome that are missing from the SPAdes assembly.

The GO analysis for each of the assemblies shows the MIRA assembly has on average twice much as information in terms of genes assigned to cellular component, molecular function and biological processes. For both assemblies, the most representation were for molecular function in the categories of binding (1,169 and 2,407 for SPAdes and MIRA, respectively) and catalytic activity (907 and 1,891 for SPAdes and MIRA, respectively); for biological process in the categories of cellular process (540 and 1,176 for SPAdes and MIRA, respectively) and regulation (231 and 467 for
SPAdes and MIRA, respectively); and for cellular component in the categories of nucleus (167 and 342 for SPAdes and MIRA, respectively) and cytoskeleton (94 and 180 for SPAdes and MIRA, respectively).

\subsubsection{Previously Identified Genes}

As mentioned before, only a few proteins have been characterized for L. pectinata. In terms of gene sequence, the gene structure for $\mathrm{HbI}$ from $L$. pectinata is partially characterized, while the genes coding for $\mathrm{HbII}$ and $\mathrm{HbIII}$ are fully characterized having a 4exon/3intron gene structure [30]. We wanted to find the complete $\mathrm{HbI}$ gene sequence in both of our draft genome assemblies. For this, we performed searches with the BLAST tool [31]-[33], using BLASTN optimized for somewhat similar sequences, using the $\mathrm{HbI}$ incomplete gene sequence, the HbII gene and $\mathrm{HbIII}$ gene sequences.

With the MIRA assembly, the search with the HbI incomplete gene sequence generated 2,456 hits, none of these contigs add more information to the $\mathrm{HbI}$ gene sequence. Of all of these, three matches had an alignment of more than $500 \mathrm{bp}$ with identities above $98 \%$, covering part of the first incomplete intron an exon 2 , the end of intron 2, exon 3-intron 3, and intron 3 and most of exon 4. Most of the sequences obtained with the Blastn results using the $\mathrm{HbI}$ incomplete gene sequence as query, align with the regions of $\mathrm{HbI}$ where repetitive regions are found [30]. Similar searches were performed with the sequences of the HbII and HbIII genes. The HbII gene sequence produced 732 hits, of these, only five sequences had alignments of more than $300 \mathrm{bp}$ and identities higher than $95 \%$. For the HbIII gene sequence, a total of 279 hits were obtained and the complete gene was contained within two contigs with identities of $99 \%$ and $94 \%$.

For the SPAdes assembly the $\mathrm{HbI}$ incomplete gene sequence produced 4212 hits. Of all of these, only 1 contig had a length of $956 \mathrm{bp}$ that aligned to the third intron of HbI gene with $96 \%$ of identity. The rest of hits with $>90 \%$ identity aligned at repetitive sequences located at intronic regions of $\mathrm{HbI}$ sequence, these were short alignments (>123 bp). The HbII gene sequence produced 1874 hits. The longest contigs with more than $90 \%$ of identity were two fragments that only cover the first $627 \mathrm{bp}$ of this gene. The rest of the sequences with $>90 \%$ were shorter than $250 \mathrm{bp}$ and hit intronic regions known to have repetitive sequences of the HbII gene. The HbIII gene sequence produced 554 hits and the complete gene was roughly covered (a fragment by 4 contigs with identities over $94 \%$ with minimal or no overlapping).

In general, most of the sequences found performing BLASTN searches using the $\mathrm{HbI}$ incomplete gene sequence as query were sequences that aligned with the repetitive regions that we identified in the introns of $\mathrm{HbI}$ gene. The reason why there was not a unique contig that aligns with the known $\mathrm{HbI}$ gene sequence is because joining contigs at repetitive sites of a genome is a difficult decision for any assembler, and particularly problematic when using single-end reads. A similar scenario is observed for HbII which also have several repetitive regions in all of its introns. In the case of HbIII, we found longer contigs covering most of its gene sequence. However, these results suggest that MIRA produced a more adequate assembly for the L. pectinata whole genome data since it produced longer contigs that cover more of the known Hbs gene regions. 


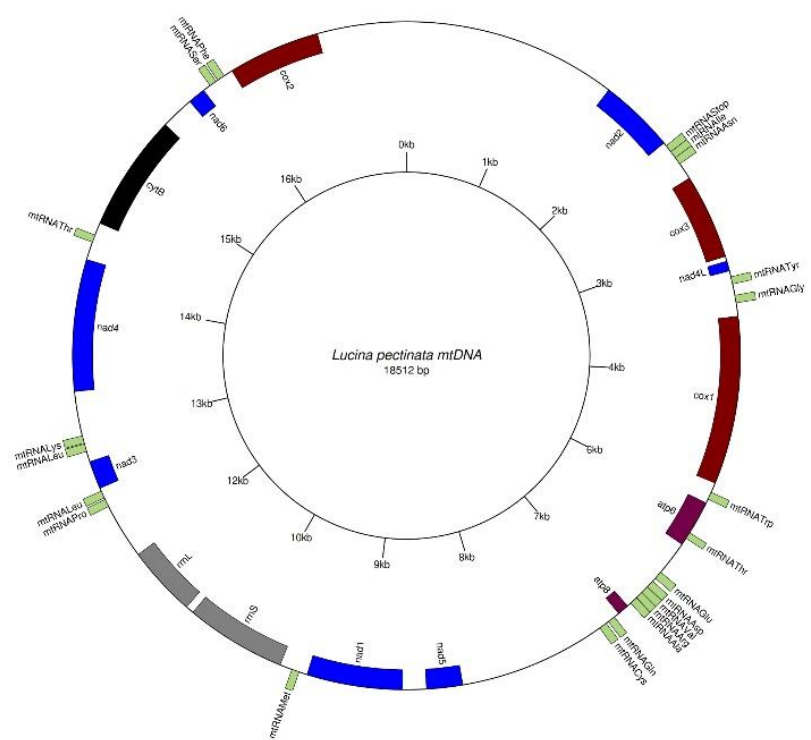

Figure 4. Visualization of the 13 mitochondrial proteincoding genes, 22 tRNAs and 2 rRNAs according to contigs found in both draft assemblies of Lucina pectinata. Genes located on the external side of the map are on "+" strand, whereas genes on the internal side are on the "-" strand.

\subsubsection{Mitochondrial Genes}

Animal mitochondrial DNA (mtDNA) is mostly a single circular molecule between 14 and $18 \mathrm{~kb}$ in length that contains a uniform set of 37 genes: 13 protein-coding genes, 2 ribosomal RNAs (rRNAs), and 22 transfer RNAs (tRNAs) [34]-[36]. With a few exceptions, most mitochondrial genomes contain no introns, having only a few intervening nucleotide pairs [35]. To locate scaffolds containing the mitochondrial genome, we selected the 13 mitochondrial protein sequences from the bivalve Solemya velum ( $S$. velum) as queries to use against the assemblies with TBLASTN. The TBLASTN search with the 13 protein sequences against the MIRA assembly produced significant matches to 34 different scaffolds. On the other hand, all the $S$. velum mitochondrial proteins sequences were found within just two SPAdes scaffolds (a 15,642 nucleotide contig, and a 2,925 nucleotide contig). Re-scaffolding of the 34 MIRA scaffolds with CAP3 yielded two larger scaffolds and re-scaffolding of the SPAdes scaffolds yielded a single scaffold. From these final scaffolds, we performed tRNA predictions. According to these assemblies the approximate size of the L. pectinata mtDNA genome is $18,512 \mathrm{bp}$ long and contains the 13 standard mitochondrial protein-coding genes: Cytochrome b (cytb), subunit I-III of cytochrome c oxidase (cox, 1, 2 and 3), subunits 6 and 8 of the $\mathrm{F}_{0}$ ATP synthetase complex (atp6 and atp8) and subunits 1-6 and $4 \mathrm{~L}$ of the respiratory chain $\mathrm{NADH}$ dehydrogenase (nad1-nad6 and nad4L). The ARAGORN program predicted 22 tRNAs in the SPAdes scaffold including two leucineencoding tRNAs and two threonine-encoding tRNAs. The INFERNAL cmscan programs predicted 2 rRNAS, one small subunit (rrnS) and one large subunit (rrnL), in the SPAdes scaffold.
Fig.4 shows the mtDNA genome organization inferred from these scaffolded sequences.

\section{CONCLUSIONS}

The de novo assembly of Ion eukaryotic data is a challenging problem. While our results indicate that the use of additional assembly methods and perhaps using additional parameter variation can improve results, from a practical matter it is difficult to do exhaustive parameter sweeps on large-timescale runs.

The shorter run time to generate a draft assembly with Spades may open the possibility to use data from additional sequencing runs in the assembly which may improve performance. We estimate that we will need approximately $25-30 \mathrm{X}$ total coverage levels to have an assembly that is sufficiently complete to capture the majority of the genes contained within the organism. With the algorithms in MIRA being NP complete, we do not foresee the possibility of adding this much additional data and still producing a result.

Overall, we believe that the MIRA assembly produced superior results for our purposes, but the assembly is still not as highquality as we would want it to be. The MIRA assembly is more highly-fragmented and is more liberal in separating repetitive segments. However, the resolution of repetitive segments may be improved by requiring MIRA to perform additional assembly iterations albeit at a higher computational cost. The SPAdes assembly we believe performed better on the compact mitochondrial genome, but in general was too conservative overall in identifying distinct repetitive elements. Both assemblies appear to require better scaffolding. Additional sequencing will likely be necessary to get around the scaffolding limitations inherent in the assembly of single-ended short reads.

\section{ACKNOWLEDGMENTS}

This work was funded through the U.S. National Institutes of Health, National Institute of General Medical Sciences Minority Access to Research Careers Grants T36-GM-008789, T36-GM095335. It also received support from the Extreme Science and Engineering Discovery Environment (XSEDE) [14], NSF grant OCI-1053575. Specifically, it used the Blacklight [12] and Bridges [13] systems which are supported by NSF award numbers ACI1041726 and ACI-1445606 at the Pittsburgh Supercomputing Center. Any opinions, findings, and conclusions or recommendations expressed in this material are those of the author(s) and do not necessarily reflect the views of the National Science Foundation. From the UPR-Medical Sciences Campus: INBRE grant NCRR P20RR16470, RCMI program NCRR grant G12RR003051, RCMI program NIMHD grant G12MD007600 and the PRCTRC National Institute on Minority Health and Health Disparities Award Number 2U54MD007587. From UPR-Mayagüez Campus: RISE-2-BEST, NIGMS grant R25GM088023.

\section{REFERENCES}

[1] P. Nicholls, "The effect of sulphide on cytochrome aa3 Isosteric and Allosteric Shifts of tha Reduced a-peak," Biochim Biophys Acta, vol. 396, pp. 24-35, 1975. 
T. Bagarinao and R. D. Vetter, "Sulfide-hemoglobin interactions in the sulfide-tolerant salt marsh resident, the California killifish Fundulus parvipinnis," f Comp Physiol B, vol. 162, pp. 614-24, Nov. 1992.

[3] S. C. Hand and G. N. Somero, "Energy metabolism pathways of hydrothermal vent animals: adaptation to a food-rich and sulfide-rich deep-sea environment," Biol Bull, vol. 165, no. April, pp. 167-181, 1983.

[4] C. L. Van Dover, "The ecology of deep-sea hydrothermal vents," Limnol Oceanogr., vol. 46, no. 8, p. 2094, 2001.

[5] L. A. Levin, "Ecology of Cold Seep Sediments: Interactions of Fauna With Flow, Chemistry and Microbes," Ocean. Mar. Biol. An Annu. Rev., vol. 43 pp. 1-46, 2005.

[6] M. R. Fisher and S. C. Hand, "Chemoautotrophic Symbionts in the Bivalve Lucina floridana from Seagrass Beds,” Biol. Bull., vol. 167, no. 2, p. 445, Oct. 1984.

[7] J. M. Rothberg, W. Hinz, T. M. Rearick, J. Schultz, W. Mileski, M. Davey, J. H. Leamon, K. Johnson, M. J. Milgrew, M. Edwards, J. Hoon, J. F. Simons, D. Marran, J. W. Myers, J. F. Davidson, A. Branting, J. R. Nobile, B. P. Puc D. Light, T. A. Clark, M. Huber, J. T. Branciforte, I. B. Stoner, S. E. Cawley, M. Lyons, Y. Fu, N. Homer, M. Sedova, X. Miao, B. Reed, J. Sabina, E. Feierstein, M. Schorn, M. Alanjary, E. Dimalanta, D. Dressman, R. Kasinskas, T. Sokolsky, J. A. Fidanza, E. Namsaraev, K. J. McKernan, A. Williams, G. T. Roth, and J. Bustillo, "An integrated semiconductor device enabling non-optical genome sequencing,” Nature, vol. 475, no. 7356, pp. 348-352, 2011

L. Liu, Y. Li, S. Li, N. Hu, Y. He, R. Pong, D. Lin, L. Lu, and M. Law, "Comparison of next-generation sequencing systems.," F. Biomed Biotechnol., vol. 2012, p. 251364, Jan. 2012.

J. Zhang, R. Chiodini, A. Badr, and G. Zhang, "The impact of nextgeneration sequencing on genomics," f Genet Genomics, vol. 38, no. 3, pp 95-109, 2011.

[10] E. J. Fox, K. S. Reid-Bayliss, M. J. Emond, and L. a Loeb, "Accuracy of Next Generation Sequencing Platforms.," Next Gener. Seq. Appl., vol. 1, no. 1, pp. 1-4, 2014.

[11] Y. Iwasaki, I. Nishiki, Y. Nakamura, M. Yasuike, W. Kai, K. Nomura, K. Yoshida, Y. Nomura, A. Fujiwara, T. Kobayashi, and M. Ototake, "Effective de novo assembly of fish genome using haploid larvae," Gene, vol. 576, no. 2 , pp. $644-649,2016$

[12] N. Nystrom, J. Welling, P. Blood, and E. L. Goh, "Blacklight: Coherent Shared Memory for Enabling Science.," in Contemporary High Performance Computing: From Petascale Toward Exascale, CRC 2013., J. S. Vetter, Ed. Chapman and Hall, 2013, p. 431-450.

[13] N. A. Nystrom, M. J. Levine, R. Z. Roskies, and J. Scott, "Bridges: a uniquely flexible HPC resource for new communities and data analytics," in Proceedings of the 2015 XSEDE Conference: Scientific Advancements Enabled by Enhanced Cyberinfrastructure, 2015, p. 30.

14] J. Towns, T. Cockerill, M. Dahan, I. Foster, K. Gaither, A. Grimshaw, V. Hazlewood, S. Lathrop, D. Lifka, G. D. Peterson, R. Roskies, J. R. Scott, and N. Wilkens-Diehr, "XSEDE: Accelerating scientific discovery," Comput. Sci. Eng., vol. 16, no. 5, pp. 62-74, 2014.

[15] S. Andrews, "FastQC: A quality control tool for high throughput sequence data.," Http://Www.Bioinformatics.Babraham.Ac.Uk/Projects/Fastqc/, 2010.

[16] B. Chevreux, T. Wetter, and S. Suhai, "Genome Sequence Assembly Using Trace Signals and Additional Sequence Information," Comput. Sci. Biol. Proc. Ger. Conf. Bioinforma., no. 1995, pp. 45-56., 1999.

[17] A. Bankevich, S. Nurk, D. Antipov, A. A. Gurevich, M. Dvorkin, A. S Kulikov, V. M. Lesin, S. I. Nikolenko, S. Pham, A. D. Prjibelski, A. V. Pyshkin, A. V. Sirotkin, N. Vyahhi, G. Tesler, M. a. Alekseyev, and P. a. Pevzner, "SPAdes: A New Genome Assembly Algorithm and Its Applications to Single-Cell Sequencing,” f. Comput. Biol., vol. 19, no. 5, pp. $455-477,2012$

[18] W. H. Majoros, M. Pertea, and S. L. Salzberg, "TigrScan and GlimmerHMM: Two open source ab initio eukaryotic gene-finders," Bioinformatics, vol. 20, no. 16, pp. 2878-2879, 2004.

[19] UniProt-Consortium, "UniProt: the universal protein knowledgebase," Nucleic Acids Res, vol. 45, no. D1, pp. D158-D169, 2017.

[20] S. D. Ghiassian, J. Menche, and A. L. Barabási, "A DIseAse MOdule Detection (DIAMOnD) Algorithm Derived from a Systematic Analysis of Connectivity Patterns of Disease Proteins in the Human Interactome," PLoS Comput. Biol., vol. 11, no. 4, pp. 1-21, 2015.

[21] V. N. Bhatia, D. H. Perlman, C. E. Costello, and M. E. McComb, "Software tool for researching annotations of proteins: Open-source protein annotation software with data visualization," Anal. Chem., vol. 81, no. 23 , pp. 9819-9823, 2009 .

[22] X. Huang and A. Madan, "CAP3: A DNA sequence assembly program.," Genome Res., vol. 9, no. 9, pp. 868-877, 1999.

[23] D. Laslett and B. Canback, "ARAGORN, a program to detect tRNA genes and tmRNA genes in nucleotide sequences," Nucleic Acids Res., vol. 32, no. 1, pp. 11-16, 2004

[24] E. P. Nawrocki and S. R. Eddy, "Infernal 1.1: 100-fold faster RNA homology searches," Bioinformatics, vol. 29, no. 22, pp. 2933-2935, 2013.

[25] N. J. Loman, R. V Misra, T. J. Dallman, C. Constantinidou, S. E. Gharbia, J. Wain, and M. J. Pallen, "Performance comparison of benchtop highthroughput sequencing platforms.," Nat. Biotechnol., vol. 30, no. 5, pp. 434-9, 2012.

[26] M. Yandell and D. Ence, "A beginner's guide to eukaryotic genome annotation," Nat Rev Genet, vol. 13, no. 5, pp. 329-342, 2012

[27] Life Technologies, "De novo assembly using Ion semiconductor sequencing," Ion Torrent Seq. All, no. Figure 1, pp. 1-4, 2013

[28] Illiumina, “De Novo Assembly Using Illumina Reads," Illumina Tech. Note Seq., 2010.

[29] R. Hinegardner, "Cellular DNA content of the Mollusca.," Comp. Biochem Physiol. A. Comp. Physiol., vol. 47, no. 2, pp. 447-460, Feb. 1974

[30] I. M. Montes-Rodríguez, L. E. Rivera, J. López-Garriga, and C. L. Cadilla "Characterization and expression of the Lucina pectinata oxygen and sulfide binding hemoglobin genes," PLoS One, vol. 11, no. 1, pp. 1-31, 2016.

[31] S. F. Altschul, W. Gish, W. Miller, E. W. Myers, and D. J. Lipman, "Basic Local Alignment Search Tool," $7 \mathrm{Mol}$ Biol, vol. 215, no. 3, pp. 403-410, 1990

[32] S. F. Altschul, T. L. Madden, A. A. Schäffer, J. Zhang, Z. Zhang, W. Miller and D. J. Lipman, "Gapped BLAST and PSI-BLAST: a new generation of protein database search programs," Nucleic Acids Res, vol. 25, no. 17, pp. 3389-3402, 1997.

[33] M. Johnson, I. Zaretskaya, Y. Raytselis, Y. Merezhuk, S. McGinnis, and T. L. Madden, "NCBI BLAST: a better web interface.," Nucleic Acids Res., vol. 36, pp. W5-W9, Jul. 2008.

[34] J. L. Boore, "Animal mitochondrial genomes," Nucleic Acids Res., vol. 27, no. 8, pp. 1767-1780, 1999 .

[35] C. T. Beagley, R. Okimoto, and D. R. Wolstenholme, "The mitochondria genome of the sea anemone Metridium senile (Cnidaria): Introns, a paucity of tRNA genes, and a near-standard genetic code," Genetics, vol. 148, no. 3, pp. 1091-1108, 1998 . goes," Trends in Genetics, vol. 19, no. 12. pp. 709-716, 2003. 